\title{
NanoTherm Therapy
}

National Cancer Institute

\section{Source}

National Cancer Institute. NanoTherm Therapy. NCI Thesaurus. Code C160824.

A procedure for the focal treatment of solid tumors. Magnetic nanoparticles of iron oxide are introduced either directly into the tumor or into the resection cavity wall. These particles are subjected to an alternating magnetic field that causes the particles to generate heat and kill the cancer cells by thermal ablation. 